Original Research Paper

\title{
Medical Images Registration based on Normalized Dissimilarity Index
}

\author{
${ }^{1}$ Amina KHARBACH, ${ }^{2}$ Mouad EL OMARI, \\ ${ }^{1}$ Amar MARDANI, ${ }^{1}$ Benaissa BELLACH and ${ }^{1}$ Mohammed RAHMOUN \\ ${ }^{1}$ LSE2I Laboratory, ENSAO, Oujda, Morocco \\ ${ }^{2}$ Department of Applied Mathematics and Information Systems Department, ESTO, Oujda, Morocco
}

\author{
Article history \\ Received: 17-06-2016 \\ Revised: $25-8-2017$ \\ Accepted: 22-9-2017 \\ Corresponding Author: \\ Amina KHARBACH \\ LSE2I Laboratory, ENSAO, \\ Oujda, Morocco \\ Email: a.kharbach@ump.ac.ma
}

\begin{abstract}
Image registration is an essential step in a large number of processing chains for medical images. It is used to align two images taken at different times and from different sensors as well. In this paper, we are interested in the rigid registration and similarity measures. We describe a new registration approach, based on the normalized dissimilarity index that results from the local dissimilarity map (LDP). This LDP is obtained from distance transform applied to gray-scale images, to register, undergoing a binarization. We evaluate the performance of our method compared to the classical registration measurements such as correlation and mutual information, on a medical images database. We show that the mean squared error of our approach is more accurate in comparison to the classical registration methods to which researchers still adhere. The robustness of our proposed index is validated regarding the luminance variation and the presence of "the Pepper and Salt" as much as "the Gaussian" noise.
\end{abstract}

Keywords: Image Registration, Image Processing, Dissimilarity Index, Local Dissimilarity Map, Correlation, Mutual Information, The Pepper and Salt, Gaussiannoise

\section{Introduction}

Image registration is a fundamental task in medical image processing and an important tool in different fields. The literature related to image registration has shed lights on various applications in order to follow the localization of dissimilarity between images and to analyze the objects behavior; Citation of Zitova and Flusser (2003; Maintz and Viergever, 1998; Petrou, 2004; Viergever et al., 2016).

Each year, millions of images are produced to establish diagnosis or synthetic analysis. They provide mostly medical information on the form and the functioning of the human body, unfortunately, all these information are difficult to objectively exploit and match. Consequently, the registration provides through two approaches an alternative to overcome this scientific obstacle.

The first general approach consists in extracting image characteristics in order to register them by a similarity measure. Another method makes it possible to carry out a direct comparison of images in particular for the binary case.
In this study, we try to introduce a novel registration measure that takes account of local information on an image. The idea is emanated by the fact that classical similarity measurements of registration are satisfied to treat either the binary images or grayscale ones; Woods et al. (1992; Hill et al., 1993; Van den Elsen et al., 1995; Collignon et al., 1995a; 1995b Maes et al., 1997; Pluim et al., 2000). In the case of binary images these techniques are less efficient because of their lack of information that leads to a difficulty to describing and registering images.

We are interested, in this study in the rigid registration. We present a novel a registration approach based on normalized dissimilarity index that results from the Local Dissimilarity Map (LDP). Proposed by Baudrier et al. (2008), the local dissimilarity map presents a useful means to compare two images by offering a localization and a quantification of the differences, some scientific work based on this map are presented in the Citation of Fedorov et al. (2008) where the authors use it to evaluate the accuracy of image registration; and in the citation of Pogam et al. (2008) where the researches correct sampling problems in 
tomography volumes acquisitions. The cornerstone of our proposed measure is that it can be applied on both monomodal and multimodal registration types, thanks to the step of binarizing the images to be registered. The reason in adopting such approach in medical images is to estimate a geometric transformation allowing the spatial superposition of anatomical structures present in each medical image and to provide a viable system to the expert for a better vision over medical images comparison in order to verify as quickly as possible the effects of the initiated treatment and to make a more precise diagnose thus diseases.

Our paper is organized according to the different sections. The Section 2 presents registration concepts and its classical measures. The Section 3 summarizes our proposed method based on the local dissimilarity map. Finally, the discussion of the experimental results and the conclusion are respectively reported in the sections 4 to 5 .

\section{Classical Registration Measures}

Image registration is the task of finding a spatial oneto-one mapping from pixel in one image to pixel in the other image. Its framework is illustrated in "Fig. 1". In this study, we propose two images $I_{f}$ and $I_{m}$ with the same size. The first one presents the reference image and the second $I_{m}$ is the moving one; we consider $T_{\mu}$ the transformation that makes $I_{m}$ spatially aligned to $I_{f}$ with the parameter vector $\mu$ containing one rotation angle and the translations in $x$ and $y$ direction. Commonly, the registration problem is formulated as an optimization problem in which the similarity function is minimized:

$$
T_{\mu o}=\operatorname{argmin}-S\left(I_{f}, T_{\mu}\left(I_{m}\right)\right)
$$

where, $S$ is a similarity measure that defines the qualityof alignment.

After $T_{\mu 0}$ is determined, it is applied to the moving image to produce the registered moving one. This latter is then compared to the reference image in order to test if the registration process is performed.
In this study, we take an interest in the rigid registration (rotation and displacement) and more precisely in the similarity measure. Despite the existence of a panoply of the registration measures we chose the most used in literature such as the correlation and the mutual information; Citation of Lisa Brown (1992). These measures are described below:

\section{Correlation}

The maximum of Correlation function is used to determine how to align the images. Its equation is defined by:

$$
\operatorname{Corr}\left(I_{f}, I_{m}, \mu\right)=\sum_{x \in \Omega_{f}} I_{f}(x) I_{m}\left(T_{\mu}(x)\right)
$$

where, $\Omega_{f}$ is the domain of the fixed image.

The correlation technique is more used in the monomodal image registration where there exists linear relationship between measurements for the homologous structures of images. It varies according to the common surface between the fixed and moving images. The more this surface is large, the more the correlation will be important. However, if the images are superposed on a small surface, the correlation will be weak. In order to avoid this situation, there exists the normalization of correlation function that is given by:

$$
\operatorname{CorrN}\left(I_{f}, I_{m}, \mu\right)=\frac{\sum_{x \in \Omega_{f}} I_{f}{ }^{\prime}(x) I_{m}{ }^{\prime}\left(T_{\mu}(x)\right)}{\sqrt{\sum_{x \in \Omega_{f}}\left(\left(I_{f}{ }^{\prime}(x)\right)^{2} \sum_{x \in \Omega_{f}}\left(I_{m}{ }^{\prime}\left(T_{\mu}(x)\right)\right)^{2}\right.}}
$$

Where:

$$
\begin{aligned}
& I_{f}^{\prime}(x)=I_{f}(x)-\overline{I_{f}(x)} \\
& I_{m}^{\prime}(T \mu(x))=I_{m}(T \mu(x))-\overline{I_{m}(T \mu(x))}
\end{aligned}
$$

$\overline{I_{f}(x)}$ respectively $\overline{I_{m}(T \mu(x))}$ are average values of two images.

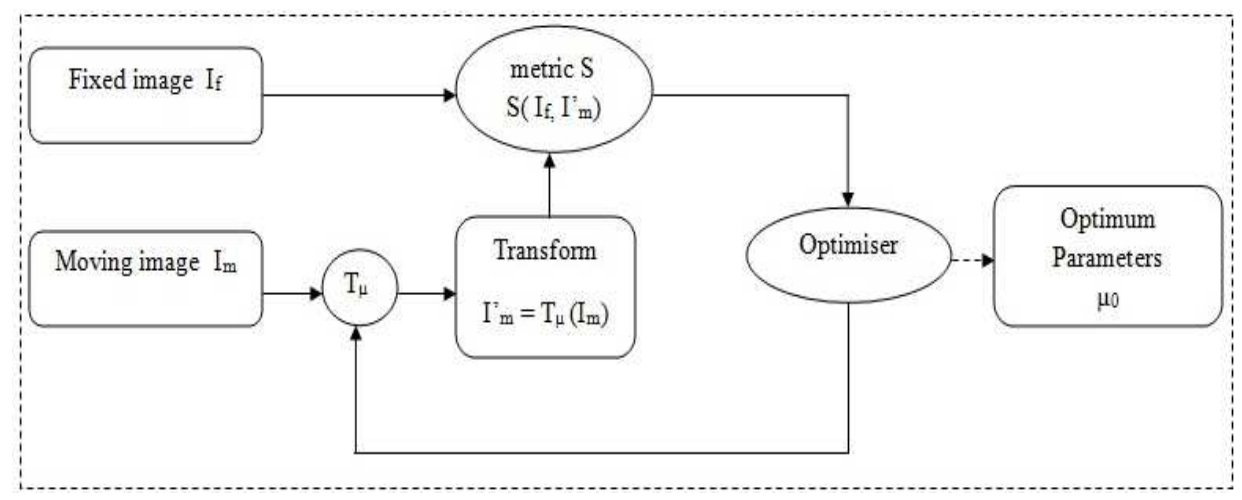

Fig. 1. The basic registration components 
Although the correlation method is faster and easier to implement in real time at the analysis system, it is unfortunately sensitive to the intensity changes due to the noise, the luminance variation and the use of many sensors.

\section{Mutual Information}

Mutual information is inspired by the information theory. It is appropriate to match images taken from many acquisition sources without applying the segmentation step.

Thus, transforming an image more similar to the second one can be done by maximizing their mutual information. It is defined as follows:

$$
M I\left(I_{f}, I_{m}\right)=H\left(I_{f}\right)+H\left(I_{m}\right)-H\left(I_{f}, I_{m}\right)
$$

where, $H\left(I_{f}\right)$ (respectively $H\left(I_{m}\right)$ ) presents the entropy measure which describes the amount of information of fixed and moving images.

It is expressed by:

$$
H\left(I_{f}\right)=\sum_{a \in I_{f}} p_{a}(a) \log p_{a}(a)
$$

Respectively:

$$
H\left(I_{m}\right)=\sum_{b \in I_{m}} p_{b}(b) \log p_{b}(b)
$$

Where:

$p_{a}(a)=$ The probability that a pixel of image $I_{f}$ has the value $a$

$p_{b}(b)=$ The probability that a pixel of image $I_{m}$ has the value $b$

The common information of $I_{f}$ and $I_{m}$ that measures the quantity of information brought at the same time can be expressed as:

$$
H\left(I_{f}, I_{m}\right)=\sum_{a \in I_{f}} \sum_{b \in I_{m}} p_{a b}(a, b) \log p_{a b}(a, b)
$$

where, $p_{a b}(a, b)$ being the probability that a pixel of image $I_{f}$ has the value $a$ and $b$ in $I_{m}$.

The maximization of mutual information is well adapted to multimodal registration; Citation of Viola et al. (1997). Indeed, if two images represent the same anatomical structure, they will have the mutual information between them. Contrary to the correlation method which supposes that the images to be aligned have similar intensities. Its normalization is proposed by using a fraction of two terms depending on the common surface of the two images (Equation 8):

$$
\operatorname{NMI}\left(I_{f}, I_{m}\right)=\mathrm{H}\left(I_{f}\right)+\mathrm{H}\left(I_{m}\right) / \mathrm{H}\left(I_{f}, I_{m}\right)
$$

Images are identical when their mutual information reaches the maximum value. Because of the nonlinear relationship that can occur between images intensities through many modalities, the correlation measure was generally shown an unsuitable candidate for the multimodal image registration.

In this context, we propose, in the next section, a novel dissimilarity measure that we use to registering not only grayscale images but also images undergoing the binarization step.

\section{Proposed Method}

In this study, we introduce a new registration approach including the idea based on the construction of a scalar that calculates dissimilarity between images to register. We apply then an algorithm for researching transformation by minimizing this scalar. This latter is a quadratic sum of the local dissimilarity map values of $\mathrm{N}^{*} \mathrm{M}$ size that treats not only binary images but gray-scale ones.

\section{Binary Images}

The binary images cover several processes. They can be provided through binary sensors, edge extraction or a binarization step. The problem of their registration was the starting point of our work.

The proposed index is a quadratic sum of the local dissimilarity map values. This map presents an efficient way to quantify and localize differences between two images. It depends on the local information variations and it can be calculated from:

- The algorithm based on the distance transform

- The algorithm based on the Hausdorff distance

Due to the simplicity of implementation and speed of execution, we based our study on the second algorithm. Its equation is as follows:

$$
L D M=I_{f} * t d\left(I_{m}\right)+I_{m} * t d\left(I_{f}\right)
$$

where, $t d$ is the image distance transform that makes it possible to know the distance between a given pixel and the nearest one; Citation of Paglieron (1992).

The example illustrated in "Fig. 2" shows the detected differences between two binary images by applying the local dissimilarity map based on the distance transform.

Our index represents in one number value of the indicators associated with the multiple characteristics of the local dissimilarity map. For its normalization we refer to the Frobienus or Schur matrix norm defined as follows:

The Schur norm of a matrix A of size $m * n$ is an application of $M(I R)$ to IR such that:

$$
\|A\|=\sqrt{\sum_{i, j=1}^{n, m}\left|a_{i j}\right|^{2}}
$$




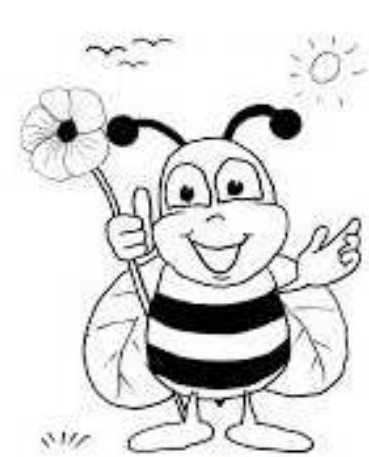

a

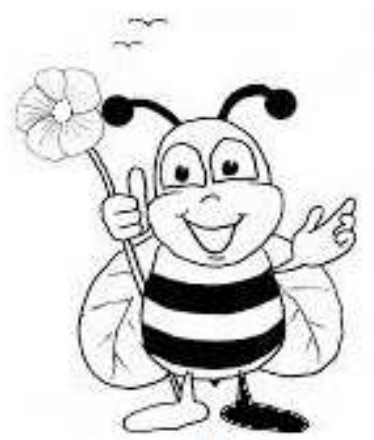

b

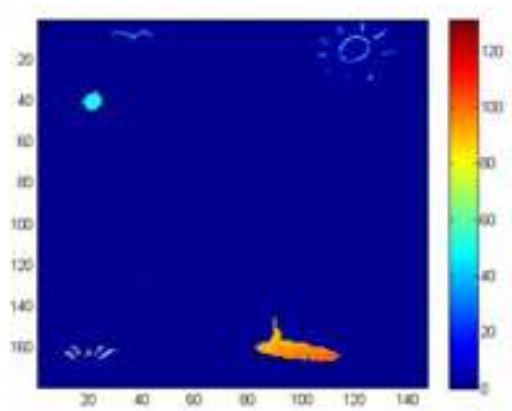

C

Fig. 2. An Example showing the calculation of difference between input images (a) and (b). (a): Image A, (b): Image B, (c): Local dissimilarity map between $\mathrm{A}$ and $\mathrm{B}$

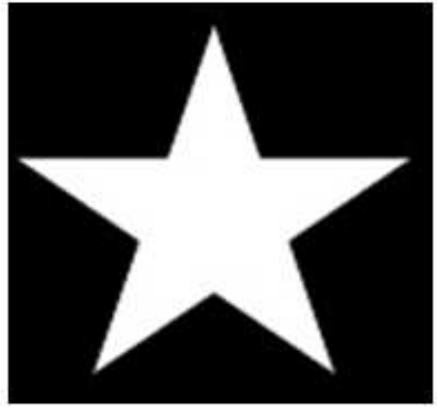

a

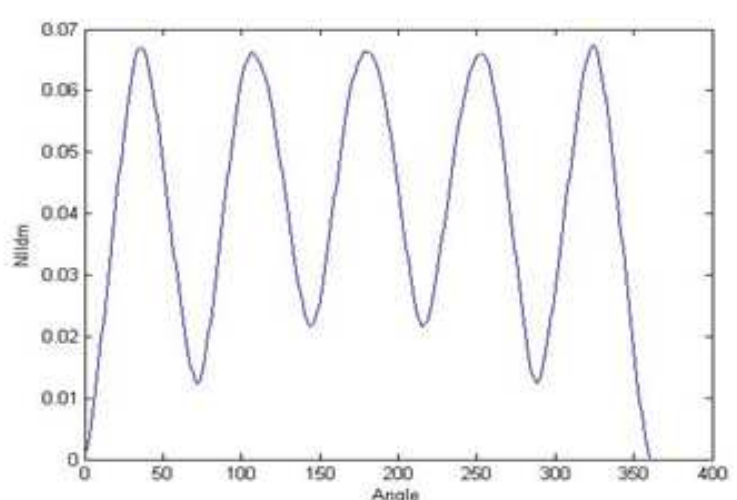

b

Fig. 3. The normalized dissimilarity index evaluation compared to the variation of rotation. (a): Binary Image (b): Illustration of the normalized dissimilarity index compared to the angle varied from $0^{\circ}$ and $360^{\circ}$

From this equation, we can define our index as:

$N I_{l d m}=\varepsilon^{*} \sqrt{\sum_{p}(\operatorname{LDM}(p))^{2}}, 0 \leq \varepsilon \leq 0.003$

where, $\varepsilon$ is the empirical parameter that depends on the size of local dissimilarity map.

The multiplication of our proposed index by the empiric value $\varepsilon$ thus causes to normalize its field of variation to the interval $[0.1]$.

In our case its minimum value is zero, this index takes the zero value when the images are identical.

The maximum value that the index can take is 1 i.e., when the difference is complete.

With the aim of closely following the evolution of our proposed index compared to the transformation and proving its efficiency for image registration. We initially considered a binary image to which we applied a series of rotation varied from $0^{\circ}$ to $360^{\circ}$.

In this example "Fig. 3", the normalized dissimilarity index corresponds to the ideal registration when it tends towards zero; this leads us to deduce that if this scalar is minimized, we will obtain a good alignment of the binary images.

\section{Gray-Scale Images}

The normalized dissimilarity index, that we have seen that it can be used to register the binary images, can be also reformulated with the distance transform extension for graysale images. Relying on the state of the art of the distance transform cited by Céline Fouard (2006), two possibilities are retained: Such as taking the sum of the pixels values along the given way that is called the Gray WEIGHTED DISTANCE TRANSFORM (GWDT); or taking the length of the given way along the defined surface in the treated image that is known by the WEIGHTED DISTANCE TRANSFORM ON CURVED SPACE (WDTOCS). In this paper, we are based on the gray WEIGHTED DISTANCE TRANSFORM algorithm because of its speed and simplicity of implementation. In an attempt to anticipate the behavior of our method based on the gray weighted distance transform for the image registration, we generated curves that are good evaluation tools. 


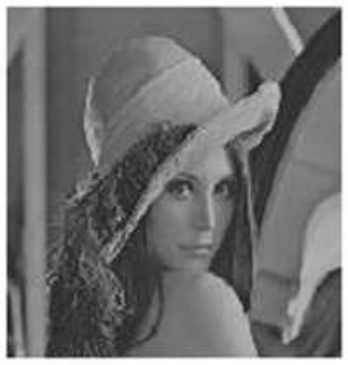

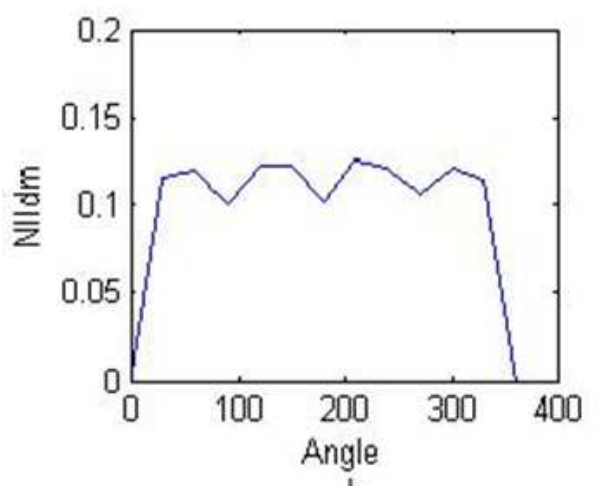

b

Fig. 4. The normalized dissimilarity index evaluation of Lena image compared to the variation of rotation. (a): Lena Image, (b): Illustration of the normalized dissimilarity index compared to the angle varied from $0^{\circ}$ and $360^{\circ}$

For that, the Lena image "Fig. 4" is aligned with the same image undergoing a series of rotations without translation. Consequently, we stress that the proposed index values, for various rotations, are very regular and do not present local minimums. Moreover, the global minimums show clearly the ideal transformation.

\section{Result and Discussion}

The validation of the registration algorithm is one of the most significant problems in particular in the medical field as it concerns the health of individuals.

In this section, we evaluate the normalized dissimilarity index robustness compared to the classical methods, we validate then the registration algorithm based on our proposed index in order to have an efficient and robust tool with a minimum of interaction with the practitioner.

\section{Validity of Proposed Index Robustness}

In this part, we evaluate the normalized dissimilarity index performance compared to the mutual information and the correlation measures by applying a series of modifications (binarization, luminance variation and noise) to models of images.

\section{Comparison between Gray Scale and Binary Images Index}

Firstly, we deprived the human brain image of great quantity of information. For that, a preprocessing step is primordial. It is a question of using image processing tools in order to purify images of all harmful defects for a good data analysis. Admittedly, we decompose, at the beginning, the reference image representing a cerebral view using the Aujol-Chambolles algorithm proposed by Aujol et al. (2006). This algorithm allows to discretize the original image in three components: Structures, textures, noise.
Their assumption is to consider noise as a distribution modelized by the Besov space cited by of Aujol and Chambolle (2005).

Secondly, a filtering step is necessary in order to highlight treated image edges. This is easily achievable by the Canny filter that allows to identify edges of studied image structure. The "Fig. 5" presents obtained results by the implementation of Aujol-Chambolles algorithm and the Canny filter.

Thirdly, we applied a translation from [-3-3] to $[+3$ $+3]$ on the original image, the results are shown on the figure "Fig. 6".

They illustrate the behavior of studied registration measures (proposed index, correlation and mutual information) compared to the translation.

These curves are of very good tool to envisage the maximum or minimum search algorithm behavior. Let us note that our proposed index behaves similarly to the other classical registration measures (mutual information and correlation function). Their minimum or maximum shows clearly the ideal alignment which responds better to our research assumption by using our approach as a registration means.

By comparing the resulting values of our alternative applied to the untreated image and that which has undergoes a preprocessing, It is noted that our proposed index behavior remains stable even if that the original image was deprived by a great quantity of information.

"Figure 7", clearly illustrates this study, which enable us to provide a minimization in computing time of the registration algorithm based on our suggested index on binary images thanks to the binarization step.

In other words, the use of the rough image is more time-consuming.

The result shows that our approach makes possible to match medical images taken from different sensors (multimodal case) using the binarization step. 


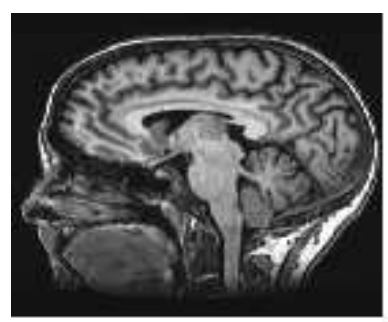

a.

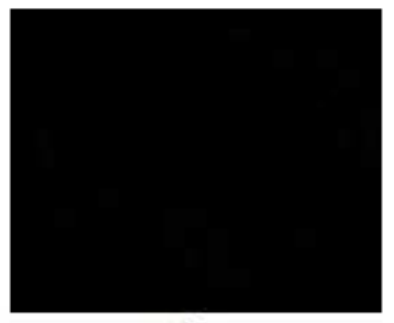

d

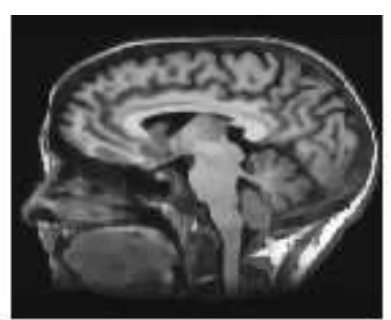

b

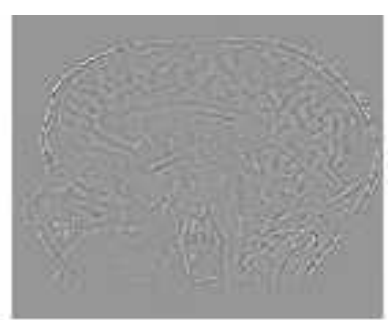

C

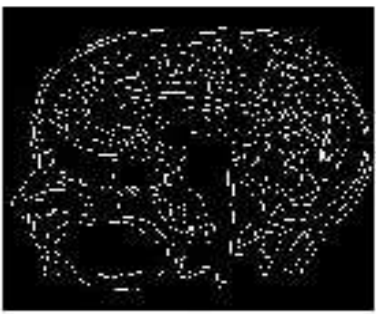

e

Fig. 5. Aujol-Chambolle's and Canny Edge detection results for human brain image. (a) ReferenceImage (b) Structure (c) Texture (d) Noise (e) Edge of structure

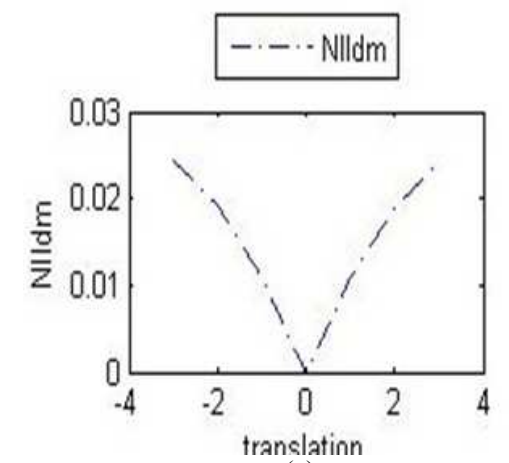

(a)

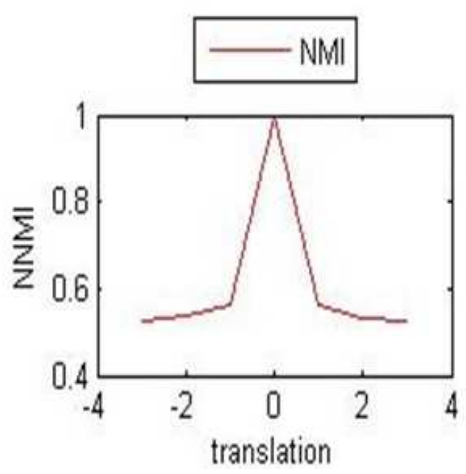

(b)

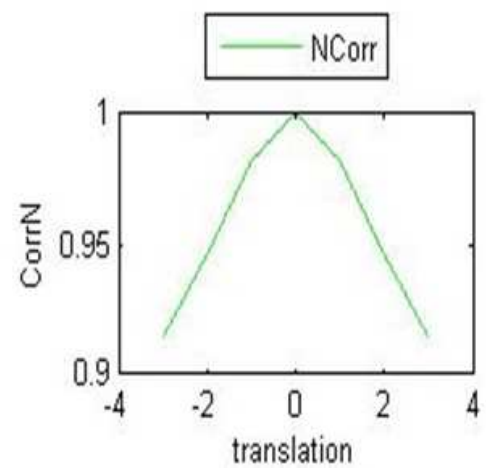

(c)

Fig. 6. The similarity/dissimilarity measurement behavior of original human brain image compared to the translation variation. (a) Normalized local dissimilarity index compared to the translation variation (b) Normalized Mutual information compared to the translation variation (c) Normalized correlation compared to the translation variation

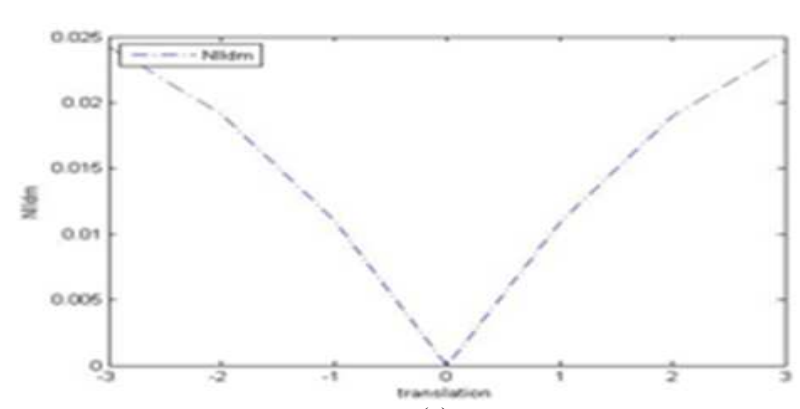

(a)

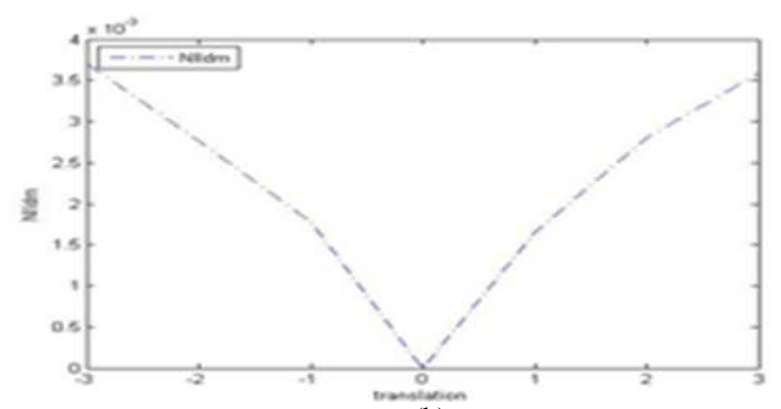

(b)

Fig. 7. Normalized dissimilarity index behavior compared to the translation variation. (a) Normalized dissimilarity index behavior of original human brain image (b) Normalized dissimilarity index behavior of binary edge structure of human brain image 


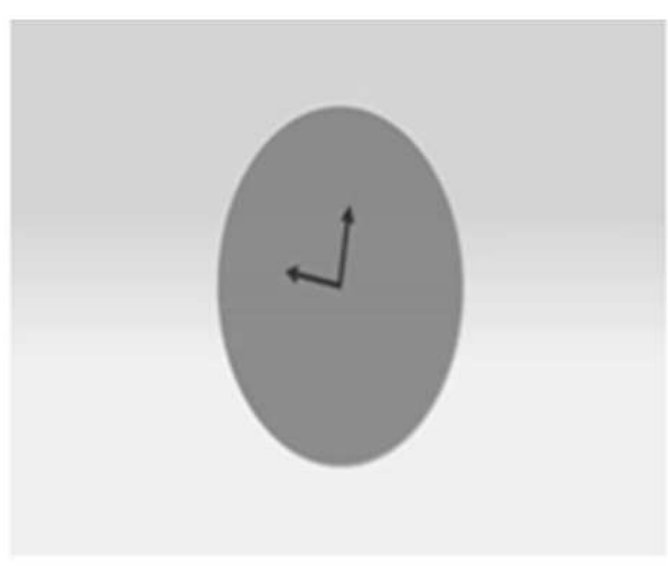

(a)

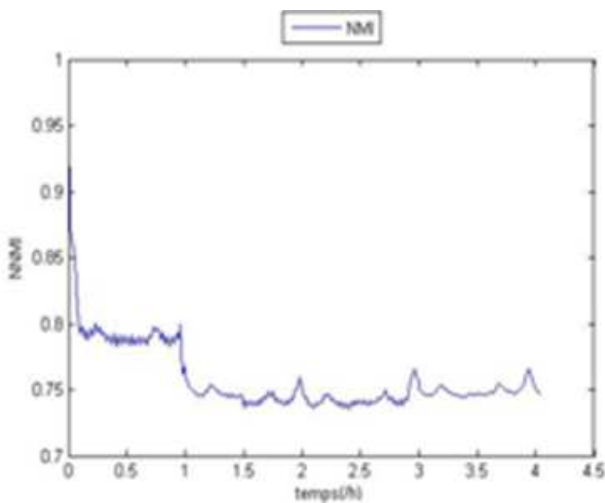

(c)

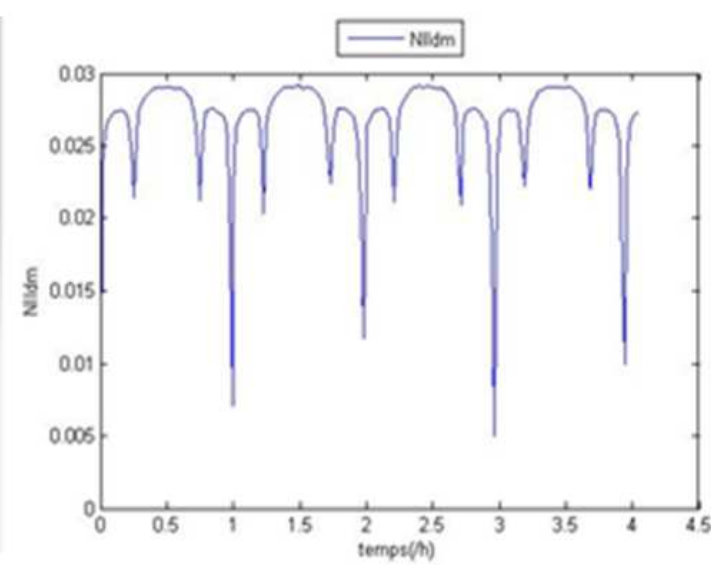

(b)

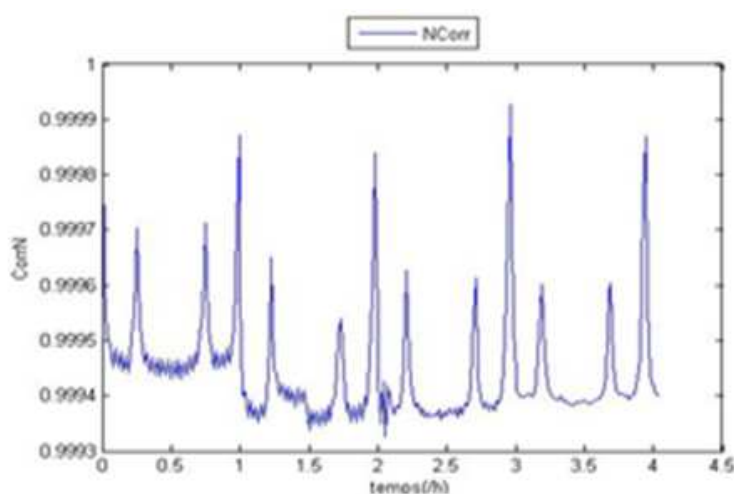

(d)

Fig. 8. The evaluation of a clock behavior by comparing different registration functions. (a) Clock example (b) Normalizeddissimilarity index curve (c) Normalized mutual information measure curve (d) Normalized correlation values compared on time

\section{Index Robustness Compared to an Object's Luminance Variation}

In this section, we generated curves in order to evaluate our method behavior and to test its efficiency compared to the similarity measures mentioned above according to two cases.

\section{Images of Identical Intensity}

In this part, we evaluated our measure compared to mutual information and correlation applied on an image database presenting the behavior of a clock during three hours.

From the figure "Fig. 8", we can note that this example shows clearly the efficiency of our approach. Indeed, the normalized dissimilarity index value is progressed in a very regular way and does not induce local minima or maxima, moreover, its minimization provides good performance in the alignment. It is also noted that the maxima of the normalized correlation index shows good alignment what shows that it remains a good choice for monomodal registration even if it presents some vibrations. However, the normalized mutual information index appears less adapted in this test.

\section{Objects of no Identical Luminance}

By varying gray tones of the circle form "Fig. 9", the correlation function shows that the result is not the same and it depends on the used tones. Its value increases with the intensity variations. This is due to the dominant force of the pale tones compared to the dark ones. Contrary to the normalized mutual information index and the proposed index that proved to be unchanged even if that we make increase the gray tones of the form. Similarly to the normalized mutual information measure, that it is already shown, in the scientific literature, a potential candidate for the multimodal registration.

This result is a good proof of the best quality of the our suggested index compared to the intensity variation and of the normalized correlation index that it is efficient only for the monomal case.

We reproduce, in this section as comparison, the curves illustrating the evolution of the three alternatives applied on two images with reversed tones. The latter have undergone a translation varied 
from $[-44]$ to $[+4+4]$. The first image shows a circle form with 233 of intensity, the second one is the same form with a reversed intensity "Fig. 10". We note that our approach maximum indicates clearly the ideal transformation.

We notice also that the mutual information function behavior signals a relative change compared to the gray tones variations of the form.

This result is very good indicator of our proposed index and the mutual information measure quality compared to the correlation that was ineffective.

Indeed, its maximum does not indicate the ideal transformation and it rather presents a maximum values ring around the ideal registration. That corresponds to measures for which the gray area overlaps the clearest one of the images to register. This test shows the difficulty with regard to the multimodal registration by maximizing the correlation. Admittedly, when the images to register do not have the identical luminance, the correlation function is not reliable.

\section{Noised Images}

In the aim of the evaluating our approach behavior to the noise, we try to register Lena image with the same one undergoing transformations. Firstly, we applied a series of rotation varied from $0^{\circ}$ to $360^{\circ}$ with steps of 30, we introduced then "Pepper and Salt" with density equal to 0.02 and "Gaussian" noise with default mean and variance equal to 0.02 .

By varying the rotation parameters, the local dissimilarity index minimum always indicates the ideal transformation. By increasing the "Pepper and Salt" noise density to 0.5 ; our measure minimum does not change its behavior "Fig. 11".

Notice also that the local dissimilarity index is assessed in a regular way in spite of the presence of the "Gaussian" noise "Fig. 12". This measurement thus appears to be rather robust with the noises in comparison to the classical registration methods.

\section{Calculation Time}

We evaluate the similarity functions runtime applied on Lena's example on which we carried on a series of rotations varied from $0^{\circ}$ to $360^{\circ}$, for several values of resolution $(256,128,64,32,16,8,4,2)$, "Fig. 13 and 14 ". We note that the runtime of the normalized correlation index is way faster; however, our approach seems giving earlier results than the running of normalized mutual information index.

\section{Validity of the Registration Algorithm}

The image registration algorithm is based on two essential points:
- The choice of the similarity or the dissimilarity measures that allows to determine the alignment degree between the reference image and the transformed one

- The optimization function of the transformation until the stop criterion is reached

In our case, we applied the local dissimilarity index as our chosen metric and we used the Powell method proposed by Powell (1964) as our optimization technique for the minimum research. This algorithm enabled us to determine quickly ideal registration and is used to find the maximum value (or the minimum value) of the transformation.

On the following example "Fig.15", the obtained result of our registration algorithm, applied to binary images, returns the parameters bellow:

$$
[d x=0 ; D y=37 ; D=0]
$$

That presents the ideal transformation.

It is evident that the reference and floating images are geometrically and spatially aligned, providing consequently a correct registration image illustrated in "Fig. 15".

The best registration was found when each translation and rotation parameters, the proposed index reaches a minimum.

The exactitude and the precision are the most important properties for the registration method. The validation of its algorithm is generally not an easy task. In our case, the validation approach is used to evaluate the performance of the registration algorithm based on our suggested index in comparison with the same algorithm based on the correlation and the mutual information functions.

Indeed, we propose to evaluate the three alternatives on the standard digital image database with and without chest lung nodules (JSRT database). This database was created by the Japanese Society of Radiological Technology (JSRT) in cooperation with the Japanese Radiological Society (JRS) in 1998. Our work consists to experiment the validity of our studied registration algorithm using medical images taken from X-ray Scanner "Fig. 16".

We calculate thus the mean square error of the three studied algorithms applying transformations varied between -30 and 30 pixels and rotations varied between -20 and $20^{\circ} \mathrm{C}$ on $153 \mathrm{X}$-ray medical images of JRST database. "Fig. 17" and "Table 1" illustrate the experimental results of the mean square error of our registration algorithm. 


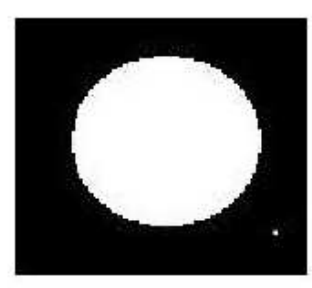

a

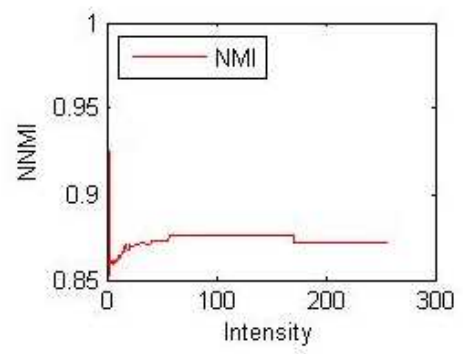

$c$

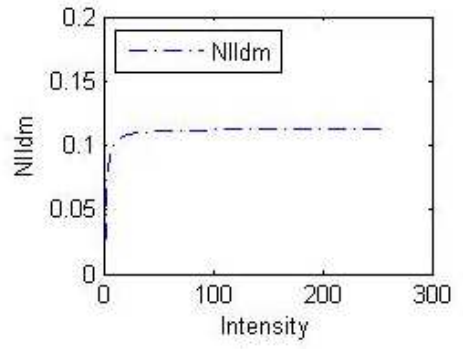

b

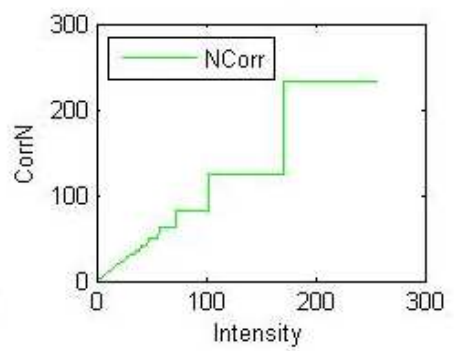

d

Fig. 9. The evolution of registration functions behavior on an image series having different gray-scales. (a): Circle image example, (b): Normalized dissimilarity index curve compared to intensity,(c):Normalized mutual information measure curve compared to intensity, (d): Normalized correlation values compared to intensity

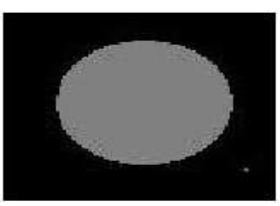

a
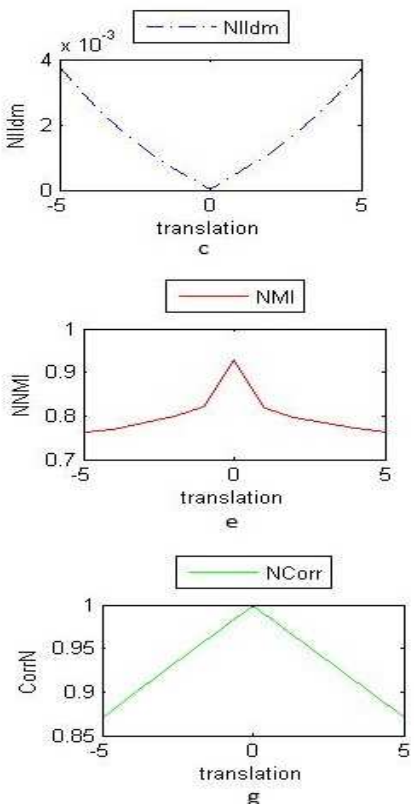

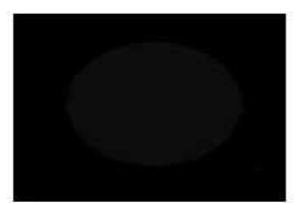

b
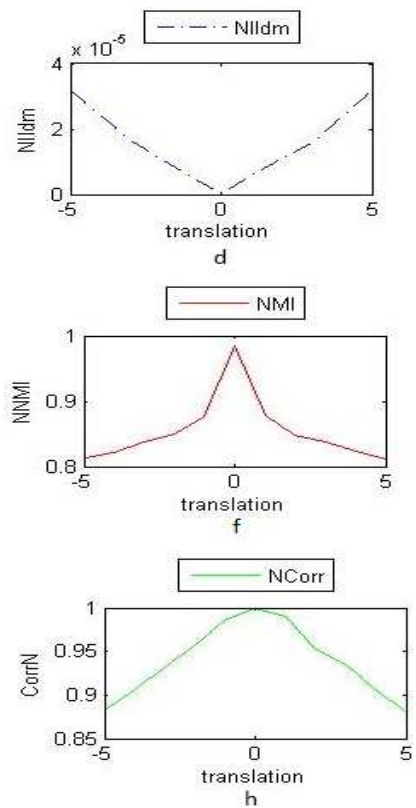

Fig. 10. The evolution of studied functions on two images with reversed gray-scale. (a): Circle with intensity $=233$, (b): Circle with intensity $=22$, (c): Normalized local dissimilarity index for image1, (d): Normalized local dissimilarity index for image 2 , (e): Normalized mutual information for image 1, (f): Normalized mutual information for image 2, (g): Normalized correlation function for image1, (h): Normalized correlation function for image 2 


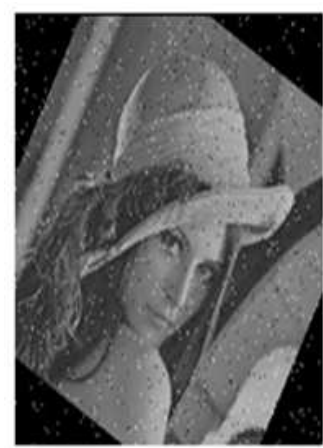

a.

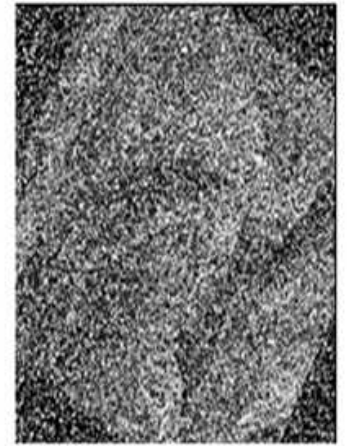

b
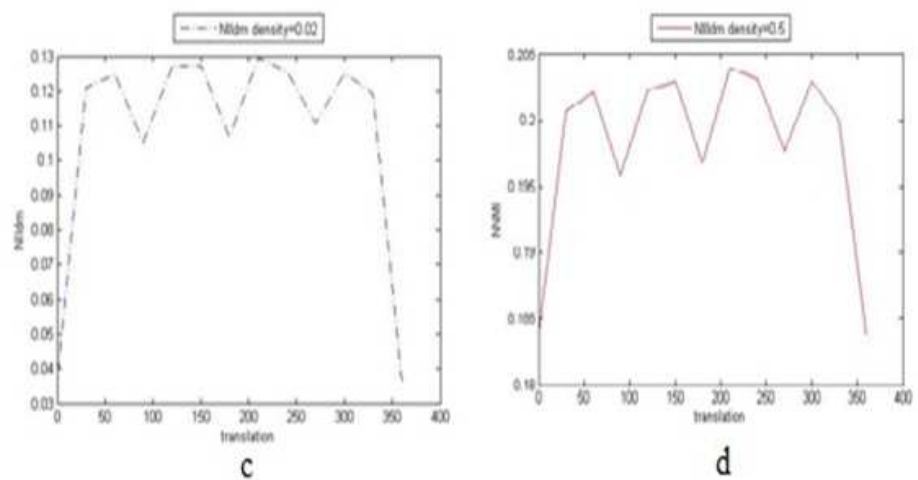

Fig. 11.The normalized dissimilarity index behavior facing "the pepper and salt" noise. (a): Lena image turned by $30^{\circ}$ with pepper and salt noise and density $=0.02$, (b): Lena image turned by $30^{\circ}$ with pepper and salt noise and density $=0.5,(\mathrm{c})$ : Normalized local dissimilarity index compared to the rotation with pepper and salt noise and density $=0.02$, (d): Normalized local dissimilarity index compared to the rotation with pepper and salt noise and density $=0.5$

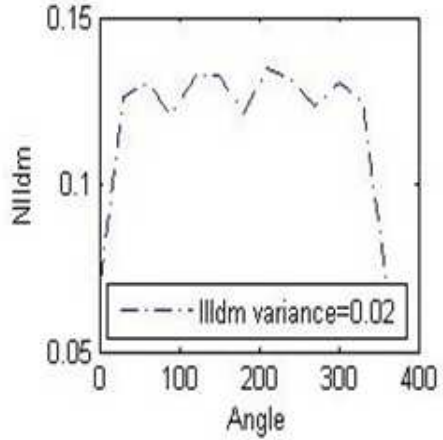

a

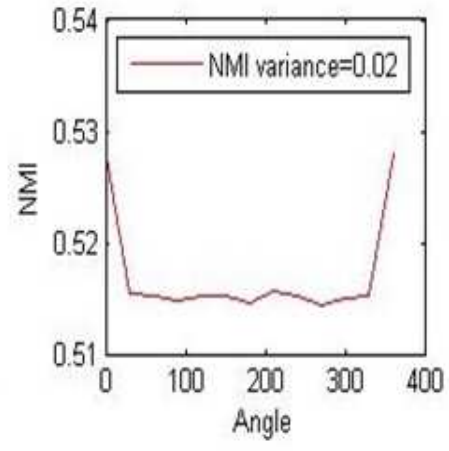

b

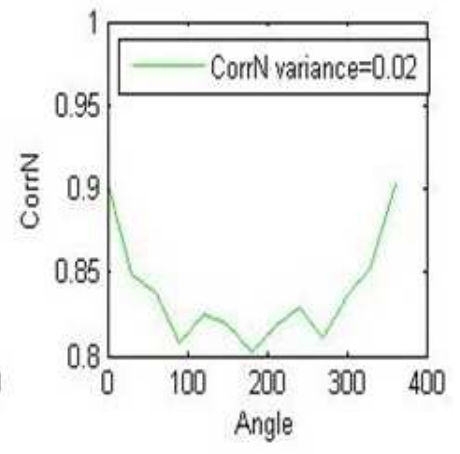

C

Fig. 12. The normalized dissimilarity index behavior facing the Gaussian noise. (a): Normalized local dissimilarity index compared to the rotation with Gaussian noise with variance $=0.02$, (b): Normalized mutual information compared to the rotation with Gaussian noise with variance $=0.02$, (c) : Normalized correlation compared to the rotation with Gaussian noise with variance $=0.02$

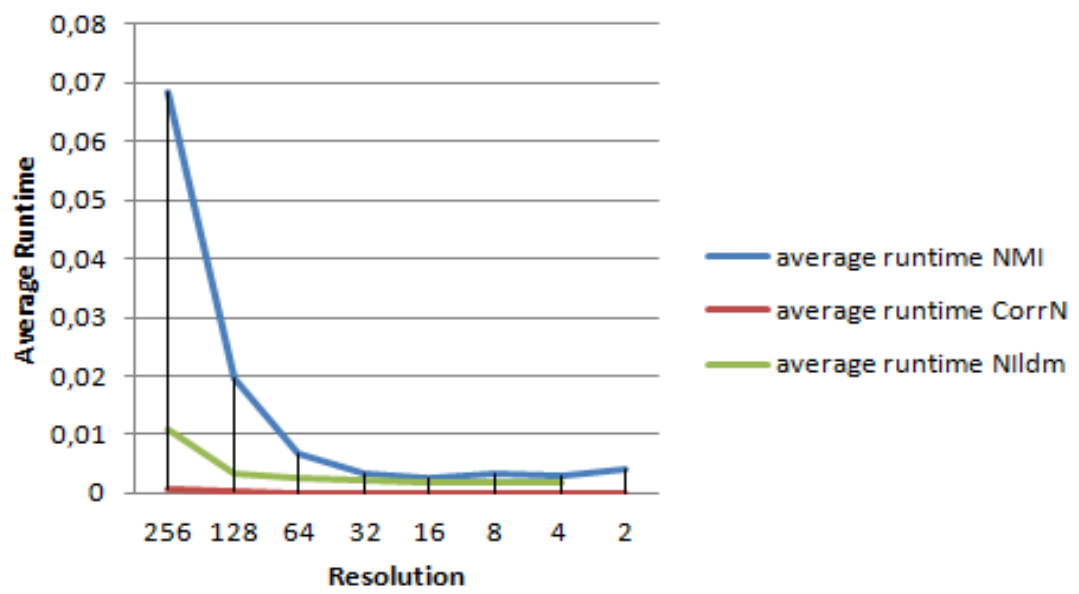

Fig. 13. The calculation time average of Similarity/Dissimilarity functions 

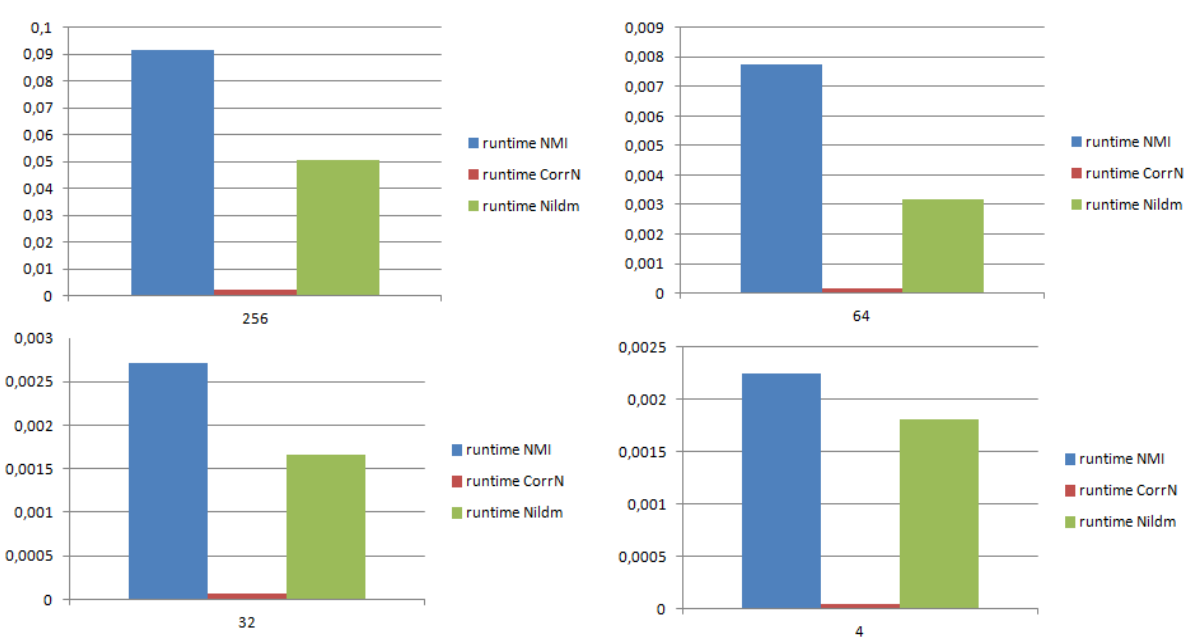

Fig. 14. Similarity/Dissimilarity functions runtime of resolution values $(256,64,32,4)$
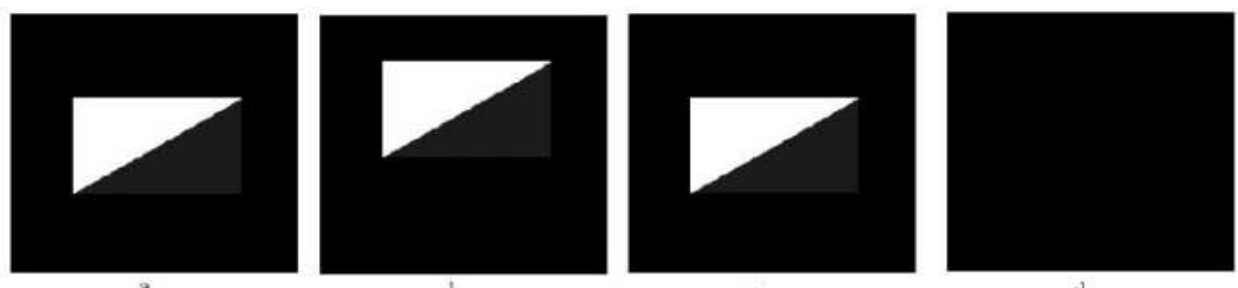

Fig. 15. Result of registration Algorithm based on the Normalized Dissimilarity Index (a) Fixed image (b) Moving image (c) Registered image (d) Difference image

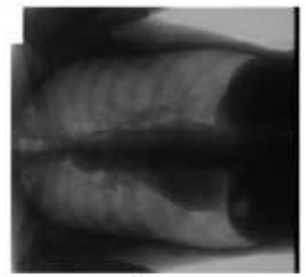

a

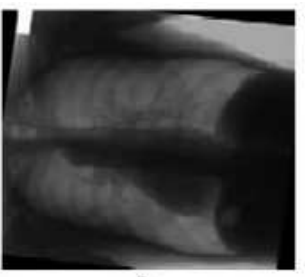

b

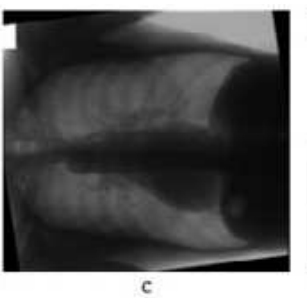

c

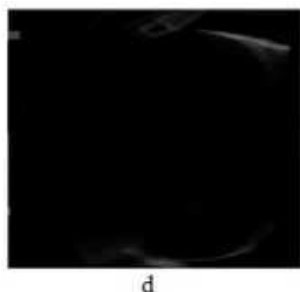

d

Fig. 16. Registration result of JSRT Image Database. (a) Reference Image (b) Transformed Image (rotation: 7, translation: 17 pixels), (c) Registered image (d) Difference between (a) and (c)

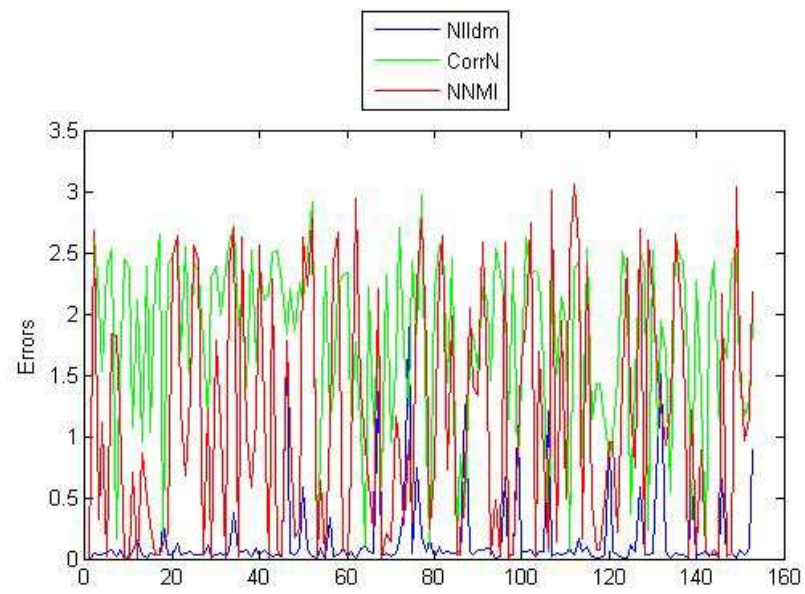

Fig. 17. The average quadratic error percentage of our registration algorithm for each studied measurement. The $\mathrm{x}$-axis presents 153 treated cases. The $\mathrm{y}$-axis presents error percentage 


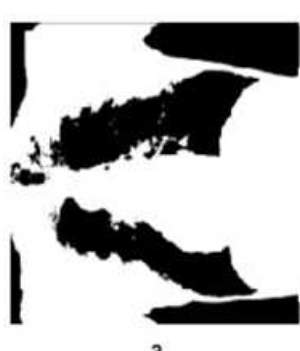

a.

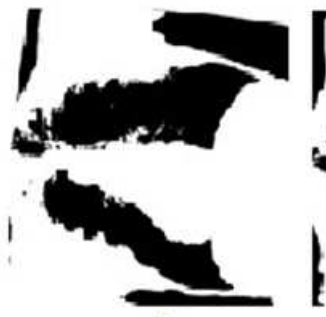

$\mathrm{b}$

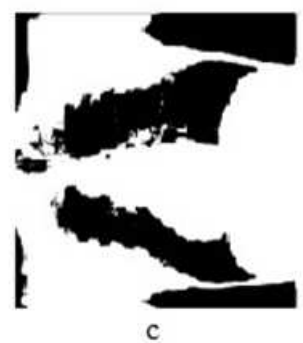

c

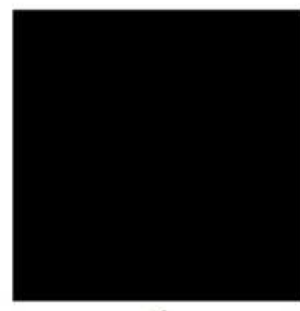

d

Fig. 18. Robust registration JSRT Image Database after binarization step. (a) Reference Image (b) Transformed Image (rotation: 7 , translation: 15 pixels) (c) Registered image (d) Difference between (a) and (c)

Table 1. Statistical measurements of the mean square error percentage of our registration algorithm for each studied measurement

\begin{tabular}{lclc}
\hline $\begin{array}{l}\text { Similarity } \\
\text { measurement }\end{array}$ & Median & Average & $\begin{array}{c}\text { Standard } \\
\text { deviation }\end{array}$ \\
\hline $\mathrm{NI}_{\mathrm{Idm}}$ & 0.1605 & 0.0472 & 0.0472 \\
$\mathrm{NI}_{\mathrm{MI}}$ & 1.7686 & 1.9565 & 0.7556 \\
$\mathrm{NI}_{\text {Corr }}$ & 1.1285 & 0.9591 & 0.9887 \\
\hline
\end{tabular}

Table 2. The mean square error percentage and the execution time of our registration algorithm for each studied measurement

Statistical

indicators

\begin{tabular}{llll} 
indicators & $\mathrm{NI}_{\text {ldm }}$ & $\mathrm{NI}_{\mathrm{MI}}$ & $\mathrm{NI}_{\text {Corr }}$ \\
\hline The mean square error & 0.0266 & 0.6808 & 0.3234 \\
The execution time & 0.003412 & 0.003643 & 0.003028 \\
\hline
\end{tabular}

Notice that the registration based on our method allows a remarkable precision compared to the classical approaches. The maximization of the correlation technique generates a good performance although it is less perform in comparison with our normalized index. Let us note also that the maximization based on the mutual information index appears less adapted in this case for this reason that it is generally used in the nonrigid and multimodal registration.

We studied a typical case among the 153 cases treated in the paper, in order to have a visibility on the timing results. We point out that we applied a series of transformations on the medical image of a patient in order to analyze the mean square error and the execution time of the applied algorithms based on the studied measures. The table below presents the results:

From the values shown in the Table 2, we can deduce that the algorithm based on our method appears more powerful compared the classical measures and faster than the normalized mutual information index.

The most important aspect of this study is to evaluate the importance of our algorithm in binary case. In this section, we present a registration example of an image sequence issued from JRST database.

Let us note that we want to reduce the information quantity of the reference image by applying a binarization step. For that, we use an adaptive and defined thresholding. We carried out the same test described previously in grayscale case. From this test

"Fig.18", we show that our technique gives satisfactory results and it is adaptable for the binary images. So our local dissimilarity index can be applied on both monomodal and multimodal registration types.

\section{Conclusion}

This work enabled us to validate the performance of our registration algorithm based on the normalized dissimilarity index compared to the mutual information and the correlation measures.

We have, in particular, studied the influence of the binarization step on our index. It is proved to be stable in spite of the image decomposition.

The results show its efficiency regarding the luminance variation and the presence of noise, compared to the classical techniques. The Normalized dissimilarity index can match not only grayscale images but also images undergoing the binarization step. Moreover, it does not require segmentation. Finally, we underline that the proposed approach is general and it can be used in multiomodal case. As perspective, we plan on proving its efficiency in no rigid registration applied on medical images taken from different sensors.

\section{Acknowledgment}

We are also immensely grateful to the reviewers comments on an earlier version of the manuscript, although any errors are our own and should not tarnish the reputations of these esteemed persons.

\section{Author's Contributions}

Amina KHARBACH: Proposed the main idea of the image registration based on on the normalized dissimilarity index. She participated in all experiments, coordinated the data analysis and contributed to the writing of the manuscript.

Mouad EL OMARI and Amar MARDANI: Participated in the experiments and they coordinated the data analysis.

Benaissa BELLACH

RAHMOUNE: Designed the research plan and organized the study. 
Mohammed RAHMOUN: Coordinated and supervised the work.

\section{Ethics}

This article is original and contains unpublished material. The corresponding author confirms that all of the other authors have read and approved the manuscript and there are no ethical issues involved.

\section{References}

Aujol, J.F. and A. Chambolle, 2005. Dual norms and image decomposition models. Int. J. Comput. Vis., 63: 85-104. DOI: 10.1007/s11263-005-4948-3

Aujol, J.F., G. Gilboa, T. Chan and S. Osher, 2006. Structure-texture image decomposition-modeling, algorithms and parameter selection. Int. J. Comput. Vis., 67: 11-136. DOI: 10.1007/s11263-006-4331-z

Baudrier, E., F. Nicolier, G. Millon and S. Ruan, 2008. Binary-image comparison with local-dissimilarity quantification. Patt. Recog., 41: 1461-1478. DOI: 10.1016/j.patcog.2007.07.011

Brown, L.G., 1992. A survey of image registration techniques. ACM Comput. Surveys, 24: 325-376. DOI: $10.1145 / 146370.146374$

Collignon, A., A. Maes, F. Delaere, D. Vandermeulen and D. Suetens et al., 1995. Automated multimodality image registration based on information theory. Inform. Process. Med. Imag., 3: 263-274.

Collignon, A., D. Vandermeulen, P. Suetens and G. Marchal, 1995. 3D multi-modality medical image registration using feature space clustering. Proceedings of the 1st International Conference on Computer Vision, Virtual Reality and Robotics in Medicine, Apr. 03-06, Springer, London, pp: 195-204.

DOI: 10.1007/978-3-540-49197-2_22

Fedorov, A., E. Billet, M. Prastawa, G. Gerig and A. Radmanesh et al., 2008. Evaluation of brain MRI alignment with the robust hausdorff distance measures. Adv. Visual Comput., 5358: 594-603. DOI: 10.1007/978-3-540-89639-5_57

Fouard, C. and M. Gedda, 2006. An objective comparison between gray weighted distance transforms and weighted distance transforms on curved spaces. Proceedings of the 13th International Conference on Discrete Geometry for Computer Imagery, Oct. 25-27, Springer, Szeged, Hungary, pp: 259-270. DOI: 10.1007/11907350_22

Hill, D., D.J. Hawkes, N.A. Harrison, N.A. and C.F. Ruff, 1993. A strategy for automated multimodality image registration incorporating anatomical knowledge and imager characteristics. Proceedings of the 13th International Conference on Information Processing in Medical Imaging, Jun. 14-18, Springer-Verlag London, pp: 182-196.

DOI: $10.1007 / \mathrm{BFb} 0013788$
Maes, F., A. Collignon, D. Vandermeulen, G. Marchal and P. Suetens, 1997. Multimodality image registration by maximization of mutual information. IEEE Trans. Med. Imag., 16: 187-198. DOI: $10.1109 / 42.563664$

Maintz, J. and M. Viergever, 1998. A survey of medical image registration. Med. Image Anal., 2: 1-36. DOI: $10.1016 / \mathrm{S} 1361-8415(01) 80026-8$

Paglieron, D.W., 1992. Distance transforms: Properties and machine vision applications. CVGIP: Graphical Models Image Process., 54: 56-74.

DOI: 10.1016/1049-9652(92)90034-U

Petrou, M., 2004. Image registration: An overview. Adv. Imag. Electron Phys., 130: 243-291. DOI: $10.1016 / \mathrm{S} 1076-5670(04) 30003-0$

Pluim, J., J. Maintz and M. Viergever, 2000. Image registration by maximization of combined mutual information and gradient information. IEEE Trans. Med. Imag., 19: 809-814. DOI: 10.1109/42.876307

Pogam, A., M. Hatt, N. Boussion, F. Turkheimer and C. Prunier et al., 2008. Conditional partial volume correction for emission tomography: A waveletbased hidden Markov model and multi-resolution approach. Proceedings of the 5th IEEE International Symposium on Biomedical Imaging: From Nano to Macro, IEEE Xplore Press, pp: 1319-1322. DOI: $10.1109 /$ ISBI.2008.4541247

Powell, M.J., 1964. An efficient method for finding the minimum of a function of several variables without calculating derivatives. Comput. J., 7: 155-162. DOI: $10.1093 /$ comjnl/7.2.155

Van den Elsen, P.A., J.A. Maintz, E.J. Pol and M.A. Viergever, 1995. Automatic registration of CT and MR brain images using correlation of geometrical features. IEEE Trans. Med. Imag., 14: 384-396. DOI: $10.1109 / 42.387719$

Viergever, M.A., J.A. Maintz, S. Klein, K. Murphy and M. Staring et al., 2016. A survey of medical image registration-under review. Med. Image Anal., 33: 140-144. DOI: 10.1016/j.media.2016.06.030

Viola, P. and W.M. Wells III, 1997. Alignment by maximization of mutual information. Int. J. Comput. Vis., 24: 137-154. DOI: 10.1023/A:1007958904918

Woods, R., S.R. Cherry and J.C. Mazziotta, 1992. Rapid automated algorithm for aligning and reslicing PET images. J. Comput. Assisted Tomography, 16: 620-633. DOI: 10.1097/00004728-199207000-00024

Zitova, B. and J. Flusser, 2003. Image registration methods: A survey. Image Vis. Comput., 21: 977-1000. DOI: $10.1016 / \mathrm{S} 0262-8856(03) 00137-9$ 